\title{
Pruritic and Pustular Eruption on the Face
}

Lara Trindade de Carvalho, MD; Leah Cohen, MD; Ameshin Moodley, MBBS

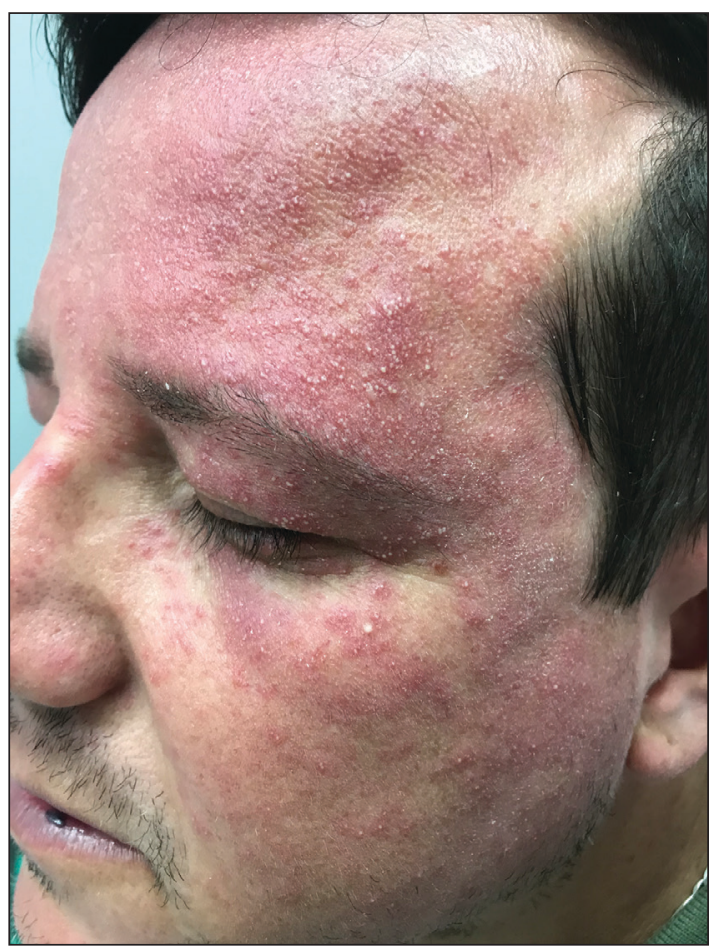

A 37-year-old man presented with a progressively pruritic and pustular eruption on the face of 2 weeks' duration. Twenty days prior to the rash onset, he began treatment for scalp psoriasis with a mixture of salicylic acid $20 \mathrm{mg} / \mathrm{mL}$ and betamethasone dipropionate $0.5 \mathrm{mg} / \mathrm{mL}$, which inadvertently extended to the facial area. One week after rash onset, he presented to the emergency department at a local hospital, where he was given intravenous hydrocortisone $100 \mathrm{mg}$ with no improvement of the rash. He had no history of skin cancer, and his family history was negative for dermatologic disease. At the time of examination, he had an erythematous eruption of follicular micropustules on the forehead, upper and lower eyelids, temples, cheeks, and mandible.

\section{WHAT'S YOUR DIAGNOSIS?}
a. demodicosis
b. eosinophilic folliculitis
c. miliaria
d. Pityrosporum folliculitis
e. rosacea

Drs. de Carvalho and Moodley are from Sinclair Dermatology, East Melbourne, Victoria, Australia. Dr. Cohen is from the Department of Dermatology, Florida International University Herbert Wertheim College of Medicine, Miami.

The authors report no conflict of interest.

Correspondence: Lara Trindade de Carvalho, MD, 1104/33 Judd Street, Melbourne, Victoria 3121, Australia

(lara.carvalho@sinclairdermatology.com.au).

doi:10.12788/cutis.0245 


\section{THE DIAGNOSIS: Demodicosis}

$\Lambda$ clinical diagnosis of facial demodicosis triggered by topical corticosteroid therapy was suspected in our patient. A 3-mm punch biopsy of the right forehead demonstrated a follicular infundibulum rich in Demodex folliculorum with a discrete mononuclear infiltrate in the dermis (Figure 1), confirming the diagnosis. The patient was prescribed metronidazole gel $2 \%$ twice daily. Complete clearance of the facial rash was observed at 1-week follow-up (Figure 2).

Demodex folliculorum is a mite that parasitizes the pilosebaceous follicles of human skin, becoming pathogenic with excessive colonization. ${ }^{1-3}$ Facial demodicosis presents as pruritic papules and pustules in a pilosebaceous distribution on the face. ${ }^{2,3}$ The diagnosis of facial demodicosis can be difficult due to similarities with many other common facial rashes such as acne, rosacea, contact dermatitis, and folliculitis.
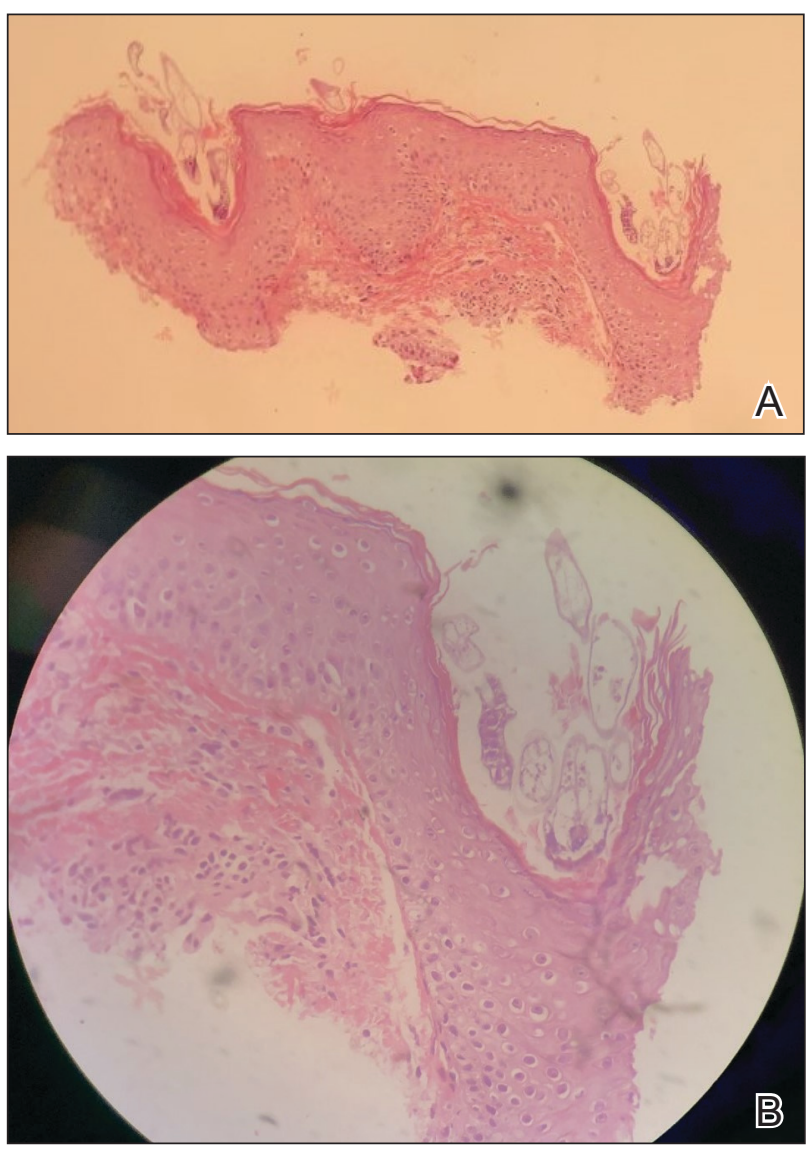

FIGURE 1. A, Two follicular infundibula infected with Demodex folliculorum with a discrete mononuclear infiltrate in the superficial dermis (H\&E, original magnification $\times 10$ ). B, Demodex folliculorum in the follicular unit $(H \& E$, original magnification $\times 40)$.
Similar to facial demodicosis, rosacea can present with erythema, papules, and pustules on the face. Patients with rosacea also have a higher prevalence and degree of Demodex mite infestation. ${ }^{4}$ However, these facial rashes can be differentiated when symptom acuity and personal and family histories are considered. In patients with a long-standing personal and/or family history of erythema, papules, and pustules, the chronicity of disease implies a diagnosis of rosacea. The acute onset of symptomatology and absence of personal or family history of rosacea in our patient favored a diagnosis of demodicosis.

Pityrosporum folliculitis is an eruption caused by Malassezia furfur, the organism implicated in tinea versicolor. It can present similarly to Demodex folliculitis with follicular papules and pustules on the forehead and back. Features that help to differentiate Pityrosporum folliculitis from Demodex folliculitis include involvement of the upper trunk and onset after antibiotic usage. ${ }^{5}$ Diagnosis of Pityrosporum folliculitis can be confirmed by potassium hydroxide preparation, which demonstrates spaghettiand-meatball-like hyphae and spores.

Eosinophilic folliculitis is a chronic sterile folliculitis that usually presents as intensely pruritic papules and pustules on the face with an eruptive onset. There are

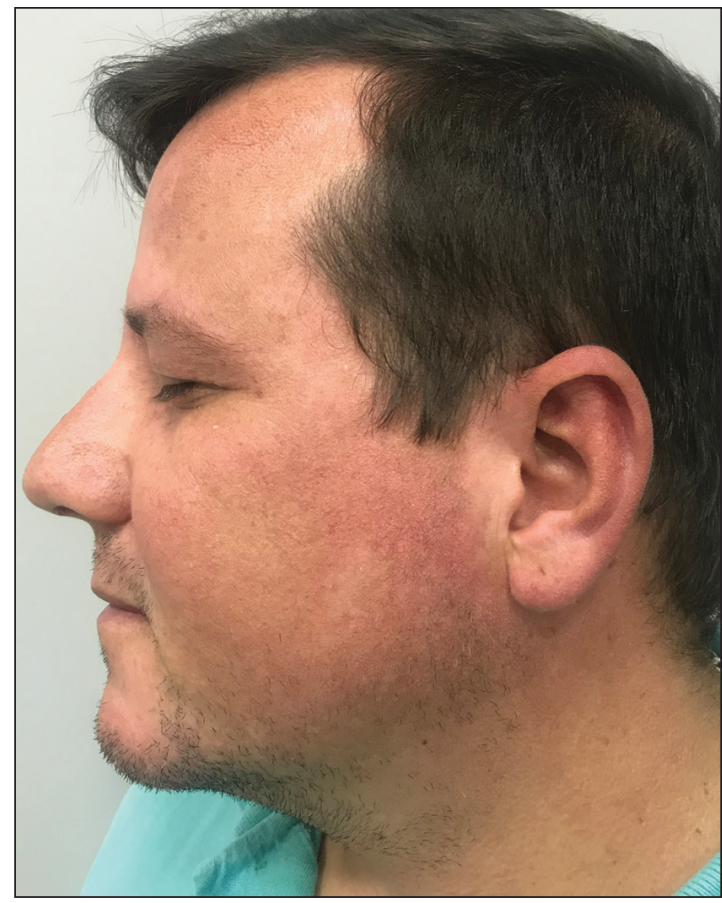

FIGURE 2. Complete resolution of the facial rash 1 week after treatment with metronidazole gel $2 \%$ twice daily. 
3 variants of disease: the classic form (also known as Ofuji disease), immunosuppression-associated disease, and infancy-associated disease. ${ }^{6}$ Eosinophilic folliculitis also may present with annular plaques and peripheral blood eosinophilia, and it is more prevalent in patients of Japanese descent. Differentiation from Demodex folliculitis can be done by histologic examination, which demonstrates spongiosis with exocytosis of eosinophils into the epithelium and a clear deficiency of infiltrating mites. ${ }^{6}$

Miliaria, also known as sweat rash, is a common condition that occurs due to occlusion of eccrine sweat glands. ${ }^{7}$ Clinically, miliaria is characterized by erythematous papules ranging from 2 to $4 \mathrm{~mm}$ in size that may be vesicular or pustular. Miliaria and demodicosis may have similar clinical presentations; however, several characteristic differences can be noted. Based on the pathophysiology, miliaria will not be folliculocentric, which differs from demodicosis. Additionally, miliaria most commonly occurs in areas of occlusion, such as in skin folds or on the trunk under tight clothing. Miliaria rarely can appear confluent and sunburnlike. ${ }^{8}$ Furthermore, miliaria is less common on the face, while demodicosis almost exclusively is found on the face.
We present the case of an otherwise healthy patient with acute onset of pruritic papules and pustules involving the superior face following topical corticosteroid use. Similar cases frequently are encountered by dermatologists and require broad differential diagnoses.

\section{REFERENCES}

1. Aylesworth R, Vance JC. Demodex folliculorum and Demodex brevis in cutaneous biopsies. J Am Acad Dermatol. 1982;7:583-589.

2. Kligman AM, Christensen MS. Demodex folliculorum: requirements for understanding its role in human skin disease. J Invest Dermatol. 2011;131:8-10.

3. Zomorodian K, Geramishoar M, Saadat F, et al. Facial demodicosis. Eur J Dermatol. 2004;14:121-122.

4. Chang YS, Huang YC. Role of Demodex mite infestation in rosacea: a systematic review and meta-analysis. I Am Acad Dermatol. 2017;77:441-447.

5. Prindaville B, Belazarian L, Levin NA, et al. Pityrosporum folliculitis: a retrospective review of 110 cases. J Am Acad Dermatol. 2018;78:511-514.

6. Lankerani L, Thompson R. Eosinophilic pustular folliculitis: case report and review of the literature. Cutis. 2010;86:190-194.

7. Hölzle ER, Kligman AM. The pathogenesis of miliaria rubra. role of the resident microflora. Br J Dermatol. 1978;99:117-137.

8. Al-Hilo MM, Al-Saedy SJ, Alwan AI. Atypical presentation of miliaria in Iraqi patients attending Al-Kindy Teaching Hospital in Baghdad: a clinical descriptive study. Am J Dermatol Venereol. 2012;1:41-46. 